\title{
Risk of Mental Disorders in Children of Parents with Alcohol or Heroin Dependence: A Controlled High-Risk Study
}

\author{
Sonia I. Vidal ${ }^{a, b} \quad$ Caroline Vandeleur ${ }^{a, b}$ Stéphane Rothen ${ }^{a, b}$ \\ Mehdi Gholam-Rezaee ${ }^{b}$ Enrique Castelao ${ }^{b}$ Olivier Halfon ${ }^{b}$ \\ Jean-Michel Aubry ${ }^{a}$ François Ferrero ${ }^{a}$ Martin Preisig ${ }^{b}$ \\ ${ }^{a}$ Department of Mental Health and Psychiatry, University Hospital of Geneva, Geneva, and \\ ${ }^{b}$ Department of Psychiatry, University Hospital Center and University of Lausanne, Prilly, Switzerland
}

\section{Key Words}

Psychopathology $\cdot$ High-risk offspring $\cdot$ Co-parent $\cdot$

Substance use disorders

\begin{abstract}
Aim: To assess the specific effect of alcohol dependence (AD) or heroin dependence (HD) in patients and their spouses on the risk of psychopathology in their 276 6.0- to 17.9year-old children (mean 11.3 years). Methods: The sample included 101 offspring of patients with AD, 23 of patients with $\mathrm{HD}$, and 152 of medical controls, as well as their 2 parents. Participants were assessed using semistructured diagnostic interviews and family history reports by psychologists blind to patient diagnoses. Results: Children of HD and AD patients had largely elevated rates of recurrent major depressive disorder. Children of HD patients were also at an increased risk for attention deficit hyperactivity disorder and substance use disorders (SUD). There were interactions between SUD in the 2 parents to increase the risk of SUD in offspring. Conclusions: These results emphasize the need for prompt identification and treatment of these children and highlight the need to pay clinical attention not only to the patient, but also to the co-parent in order to optimize prevention in offspring.

Copyright $\odot 2012$ S. Karger AG, Basel
\end{abstract}

\section{Introduction}

Excessive alcohol and illicit drug use are major public health issues. Indeed, they are associated with severe medical complications, disruption of life course, loss of productivity, and delinquency [1-8]. The familial aggregation of alcohol use disorders has been demonstrated by a large number of studies using alcoholic probands from both treatment and community settings [9-18]. Although there are fewer studies on the familiality of drug use disorders, the existing family studies also support familial aggregation of these disorders [19-21]. Therefore, the children of alcohol- and drug-dependent parents represent a population at risk for mental health problems and social malfunctioning. The study of offspring of parents with psychoactive substance dependence ('high-risk design') is a potent strategy to assess the impact of these parental psychopathologies on early psychiatric manifestations of their children [22]. The better understanding of the problems these youth experience is essential in order to promote primary and secondary prevention.

During the last two decades, twelve controlled studies of the minor offspring (in the age range between 6 and 18 years) of parents with DSM-IV alcohol or illicit drug dependence have been conducted using standardized

\section{KARGER}

Fax +4161306 1234 E-Mail karger@karger.ch www.karger.com
Sonia Vidal

Department of Psychiatry

University Hospital of Lausanne, Site de Cery

$\mathrm{CH}-1008$ Prilly (Switzerland)

Tel. +41 21643 6608,E-Mail sonia.vidal@chuv.ch 
methods including direct diagnostic interviews of parents and children and samples of at least 20 children in each group (table 1). The majority but not all of these studies found higher rates of various Axis-I disorders in high-risk offspring. Indeed, seven of the nine studies focusing on the offspring of alcoholic parents provided evidence of elevated rates of psychopathology in these children. The increased morbid risk involved substance use disorders (SUD) and disruptive behavioral disorders (DBD) in three studies as well as major depressive disorder (MDD), anxiety disorders and attention deficit hyperactivity disorder (ADHD) in two studies. Regarding the children of drugdependent parents, three [23-25] out of five studies documented increased rates of Axis-I disorders in these children. In addition, one study on offspring of parents with either alcohol or drug use disorders documented highrisk offspring to exhibit increased rates of SUD and DBD compared to children of controls [21, 23-33].

However, the large majority of studies did not take into account other factors than parental SUD that could influence the risk of psychopathology in offspring, such as the very frequent comorbid disorders in parents. Moreover, only the studies of Wilens et al. [23] and Marmorstein et al. [33] separately recruited offspring of alcohol- and drug-dependent parents, which allowed them to determine the specific effects of the two types of parental addiction by comparing the two groups of children. In the study of Hill et al. [24], which included multiplex families with high risk for alcohol dependence (AD), the effects of parental $\mathrm{AD}$ and secondary drug disorders were assessed.

Given evidence of spouse similarity for SUD $[25,34]$, the inclusion of the effect of the co-parent's SUD in the analyses is of particular relevance. However, among these twelve high-risk studies, only a small number of studies on $\mathrm{AD}$ assessed the effects of alcohol from both parents. Among them, Reich et al. [27] reported an association between the number of alcoholic parents and the risk of several specific psychiatric disorders in offspring, whereas Hill and Hruska [26] could not find such an association with respect to internalizing or externalizing disorders in offspring. Moreover, Hill and Muka [28] found an additive effect rather than an interaction between $\mathrm{AD}$ in the 2 parents regarding the risk of overall psychopathology in offspring. Using a sample from the general population, Lieb et al. [31] documented higher levels of alcohol use in children with 2 alcoholic parents as compared to those with 1 alcoholic parent, whereas the risk of alcohol abuse or dependence in children did not differ by the number of affected parents. With respect to drug disorders, only the study of Hill et al. [24] tested the effects of secondary drug disorders in both parents on child psychopathology.

Given the scarce data available on the effect of specific SUD in the 2 parents on offspring psychopathology, the goals of the present study, conducted in a European urban area, were (1) to investigate the associations between $\mathrm{AD}$ or heroin dependence (HD) in probands and Axis-I mental disorders in their offspring, adjusting for demographic variables in families and comorbid disorders in probands, and (2) to assess the effect of parental concordance for SUD on psychopathology in children.

\section{Methods}

Subjects

The data from the present study stemmed from a controlled family study. Psychiatric probands were patients with AD or HD, consecutively recruited from the inpatient and outpatient facilities of the psychiatric departments of Lausanne and Geneva, Switzerland. Inclusion criteria for the psychiatric probands were: (1) lifetime DSM-IV AD or HD, and (2) permission to enroll at least 1 of their children in the age range of 6.0-17.9 years in the study. Comparison probands were recruited from orthopedic inpatient and outpatient facilities in the same medical centers. Inclusion criteria for the comparison probands were: (1) the absence of lifetime DSM-IV AD or HD, and (2) the same criterion for inclusion of offspring as that of the AD and HD cases.

A total of 50 alcohol (participation $=63.3 \%$ ) and 15 heroin addicts (participation $=45.5 \%$ ) as well as 81 orthopedic controls (participation $=71.0 \%$ ) agreed to enroll at least 1 of their children in the required age range in the present study. Among the 15 heroin addicts, 6 also met DSM-IV lifetime dependence criteria for cocaine, 2 for hallucinogens, 4 for cannabis, and 7 for alcohol. Only 3 of them met criteria for HD alone. Probands who enrolled their children in the study did not differ from those who did not enroll their children in the study with respect to demographic variables or comorbid disorders except for ADHD. HD probands who enrolled their children in the study did not exhibit this comorbid disorder at all whereas a lifetime diagnosis of ADHD was reported by $15.2 \%$ of those HD probands who did not enroll their children in the study.

The offspring sample $(\mathrm{n}=276)$ included all of the probands' children aged between 6.0 and 17.9 years (mean 11.3, SD 3.46, 48\% girls). There were 101 offspring of probands with AD, 23 offspring of probands with $\mathrm{HD}$, and 152 offspring of comparison probands. The majority of families included $1(n=52)$ or $2(n=65)$ children, whereas 29 families had 3 or more children. Diagnostic information was also collected on 144 (91\%) of the 158 current or past partners of the probands, who were the biological co-parents of the children enrolled in the study.

\section{Procedures}

Participants were interviewed by masters-level psychologists or psychiatrists who completed intensive training over a 3-month period. Training included supervision of videotaped interviews by clinically experienced senior psychologists and each interview 
was reviewed by a senior clinical psychologist. Each family member was interviewed by a different psychologist, and interviewers and reviewers were blind to the disease status of all other members in a given family.

Diagnostic information on probands and biological co-parents was obtained using the Diagnostic Interview for Genetic Studies (DIGS) [35]. The French translation of the DIGS revealed high $\kappa$ coefficients for inter-rater reliability and slightly lower coefficients for test-retest reliability for major Axis-I diagnoses including mood [36] and SUD [37]. Offspring were directly interviewed with a French translation of the modified version of the Kiddie-Schedule for Affective Disorders and Schizophrenia (K-SADS-E) [38], which has been used in previous high-risk studies such as the Yale Family Study [21]. The K-SADS-E has been found to be a reliable and valid instrument for obtaining lifetime diagnoses in prepubertal children and adolescents $[39,40]$. Interrater reliability analyses of videotaped interviews of children using our French translation of the K-SADS-E revealed high $\kappa$ coefficients for major psychiatric diagnoses, ranging from 0.84 for depression and 0.86 for separation anxiety disorder to 1.0 for social phobia and psychosis. In addition to eliciting DSM-IV criteria for substance abuse and dependence, the K-SADS-E also elicits information on alcohol and drug consumption, defined as the consumption of alcohol or drugs at least once a week for a period of several months, without meeting criteria for abuse or dependence. Moreover, the K-SADS-E collects information on exposure to traumatic events including accidents, crimes, domestic violence and physical or sexual abuse.

Family history information was collected from all participating first-degree family members over the age of 15 years, using the Family History-Research Diagnostic Criteria (FH-RDC) [41]. The validity of the French version of the FH-RDC has previously been established by our group through the assessment of agreement between diagnoses relying on direct interviews and family history reports for a series of diagnoses in adults $[42,43]$ and children [44].

Diagnoses were assigned according to a best-estimate procedure [45], which relied on the combining of information from direct interviews, family history reports and medical records where available. Diagnoses on non-interviewed subjects were based on family history information alone. In addition to the diagnoses based on DSM-IV criteria, diagnoses of subthreshold mood syndromes in offspring were assigned using the algorithmically defined classification postulated by Angst et al. [46]. Accordingly, subthreshold depression was defined as either (recurrent) brief depression ( $\leq 5$ DSM-IV depressive symptoms for $2-$ 13 days) or minor depression (3-4 depressive symptoms for $\geq 2$ weeks). Subthreshold bipolar disorder was defined as the occurrence of hypomanic episodes without major depressive episodes, brief mania (euphoria or irritability and at least 3 or 4 manic symptoms, respectively, for 2-3 days) or minor mania (euphoria or irritability and 1-2 or 2-3 manic symptoms, respectively, for $\geq 4$ days).

Socioeconomic status was assessed using the Hollingshead scale [47], which allows for the establishment of the socioeconomic level of the family based on the professional level of each of the 2 parents.

This research project was approved by the local institutional review board. All participants gave written informed consent for their own participation prior to the assessments. In addition, parents gave written consent for the participation of their children.

\section{Data Analysis}

Between-group analyses of demographic and clinical characteristics were performed using ANOVA, $\chi^{2}$ and Fisher's exact tests as appropriate. In order to assess the associations between the proband's dependence (independent variable) and the risk of psychopathology in children (dependent variable) with adjustment for demographics variables and comorbid disorders in probands, generalized linear models were applied [48]. These models account for the lack of independence of the observations by introducing a block-diagonal exchangeable correlation structure, where each block corresponds to a given family. All analyses were also adjusted for socioeconomic status, as this variable may be associated with the child's risk of SUD [49]. Analyses were run separately for each child diagnostic outcome, and could only be applied for disorders with a lifetime prevalence of at least $5 \%$ in offspring. In a second step, drug and alcohol disorder status (abuse and dependence) of the co-parent as well as their interaction terms with the respective variable in probands were entered into the models. This enabled us to test potential interactions between the proband's and co-parent's alcohol and drug disorders (also adjusting for the co-parent's mood and anxiety disorders) on the risk of psychopathology in offspring. Additional complementary models considered the familial situation (the child living with both biological parents versus not) and the child's exposure to traumatic events as assessed in the K-SADS-E.

Statistical analyses were performed using the GENMOD procedure of the Statistical Analysis System, version 9.2.

\section{Results}

\section{Demographic Characteristics and Comorbid Disorders in Probands}

The characteristics of probands are provided in table 2. The only significant difference for demographic characteristics across proband groups was the lower proportion of married subjects among HD probands compared to the two other groups.

Regarding comorbid disorders, probands differed in the proportion of mood, anxiety, DBD and antisocial personality disorder (ASPD). Indeed, both $\mathrm{HD}$ and AD probands had increased rates of bipolar disorders (BPD), whereas only $\mathrm{AD}$ probands revealed significantly increased rates of MDD. Alcoholic probands also exhibited anxiety disorders more frequently than both heroin addicts and comparison probands, whereas DBD and ASPD were only found to be more frequent among heroin addicts compared to controls.

\section{Demographic Characteristics and Psychopathology in Co-Parents}

Diagnostic information could be collected on $86 \%$ of the biological co-parents of alcoholic probands, $81.3 \%$ of those of heroin addicts, and $96.5 \%$ of those of controls. 
Table 1. Controlled high-risk studies ( $>20$ subjects per group) of $\mathrm{HD}$ and $\mathrm{AD}$ probands with direct diagnostic interviews of offspring younger than 18 years since 1990

\begin{tabular}{|c|c|c|c|c|c|c|c|c|c|}
\hline \multirow[t]{3}{*}{ Study } & \multicolumn{5}{|c|}{ Parents } & \multicolumn{4}{|l|}{ Offspring } \\
\hline & \multicolumn{3}{|c|}{ parents with substances disorders } & \multicolumn{2}{|c|}{ comparison parents } & \multicolumn{2}{|l|}{ high-risk } & \multicolumn{2}{|c|}{ low-risk } \\
\hline & $\mathrm{n}$ & $\begin{array}{l}\text { diagnosis } \\
\text { (diagnostic system) }\end{array}$ & source & $\mathrm{n}$ & source & $\mathrm{n}$ & $\begin{array}{l}\text { mean age } \\
\text { range }\end{array}$ & $\mathrm{n}$ & $\begin{array}{l}\text { mean age } \\
\text { range }\end{array}$ \\
\hline $\begin{array}{l}\text { Hill and } \\
\text { Hruska } \\
(1992)[26]\end{array}$ & 38 & $\begin{array}{l}\mathrm{AD} \\
\text { (DSM-III) }\end{array}$ & $\begin{array}{l}\text { Members of multiplex families } \\
\text { with high risk for } \mathrm{AD} \\
\text { ( } 2 \text { brothers with } \mathrm{AD} \text { of whom } \\
\text { one was treated) }\end{array}$ & 27 & $\begin{array}{l}\text { Advertisement. } \\
2 \text { brothers with absence } \\
\text { of psychopathology. }\end{array}$ & 53 & $8-18$ & 42 & $8-18$ \\
\hline $\begin{array}{l}\text { Reich et al. } \\
\text { (1993) [27] }\end{array}$ & & $\begin{array}{l}\mathrm{AD} \\
(\mathrm{DSM}-\mathrm{III}-\mathrm{R})\end{array}$ & $\begin{array}{l}\text { Hospitalized alcoholics, } \\
\text { convicted felons, hospitalized } \\
\text { medical controls and their } \\
\text { siblings }\end{array}$ & & $\begin{array}{l}\text { Siblings of hospitalized } \\
\text { alcoholics and convicted } \\
\text { felons, hospitalized medi- } \\
\text { cal controls and their sib- } \\
\text { lings not alcoholics }\end{array}$ & $\begin{array}{l}1 \text { alcoholic } \\
\text { parent: } 82 \\
2 \text { alcoholic } \\
\text { parents: } 22\end{array}$ & $\begin{array}{l}11.5 \\
6-18 \\
14.5 \\
6-18\end{array}$ & 51 & $\begin{array}{l}11.7 \\
6-18\end{array}$ \\
\hline $\begin{array}{l}\text { Hill and Muka } \\
\text { (1996) [28] }\end{array}$ & 24 & $\begin{array}{l}\mathrm{AD} \\
\text { (DSM-III) }\end{array}$ & $\begin{array}{l}\text { Members of multiplex families } \\
\text { with high risk for AD ( } 2 \text { sisters } \\
\text { with AD of whom } 1 \text { was } \\
\text { treated) }\end{array}$ & 28 & $\begin{array}{l}2 \text { sister volunteers from } \\
\text { census. } \\
\text { No AD, drug depen- } \\
\text { dence, recurrent depres- } \\
\text { sion or schizophrenia. }\end{array}$ & 38 & $\begin{array}{l}11.3 \\
8-18\end{array}$ & 38 & $\begin{array}{l}11.3 \\
8-18\end{array}$ \\
\hline $\begin{array}{l}\text { Merikangas } \\
\text { et al. } \\
(1998)[21]\end{array}$ & 52 & $\begin{array}{l}\text { Any alcohol or drug } \\
\text { (anxiolytics, sedatives, } \\
\text { benzodiazepines or } \\
\text { marijuana) disorders } \\
\text { (DSM-III-R) }\end{array}$ & Treated subjects & 35 & $\begin{array}{l}\text { Random-digit dialing } \\
\text { procedure. } \\
\text { No psychiatric disorders. }\end{array}$ & 77 & $\begin{array}{l}12.5 \\
7-18\end{array}$ & 57 & $\begin{array}{l}12 \\
7-18\end{array}$ \\
\hline $\begin{array}{l}\text { Nunes et al. } \\
(1998)[29]\end{array}$ & $\begin{array}{l}30 \\
39\end{array}$ & $\begin{array}{l}\text { Opiate with MDD } \\
\text { (DSM-III-R) } \\
\text { Opiate without MDD }\end{array}$ & Treated subjects & $\begin{array}{l}28 \\
22\end{array}$ & $\begin{array}{l}\text { MDD, no drug } \\
\text { (DSM-III-R) } \\
\text { No MDD, no drug } \\
\text { (DSM-III-R) }\end{array}$ & $\begin{array}{l}29 \mathrm{~B} \\
19 \mathrm{G} \\
33 \mathrm{~B} \\
33 \mathrm{G}\end{array}$ & $\begin{array}{l}11.4 \\
6-17 \\
10.5 \\
6-17\end{array}$ & $\begin{array}{l}26 \mathrm{~B} \\
24 \mathrm{G} \\
18 \mathrm{~B} \\
27 \mathrm{G}\end{array}$ & $\begin{array}{l}12.7 \\
6-17 \\
13.5 \\
6-17\end{array}$ \\
\hline $\begin{array}{l}\text { Kuperman } \\
\text { et al. (1999) } \\
{[30]}\end{array}$ & 165 & $\begin{array}{l}\text { AD (DSM-III), } \\
\text { without antisocial } \\
\text { personality disorder }\end{array}$ & Treated subjects & 67 & $\begin{array}{l}\text { Community. No AD, } \\
\text { antisocial personality } \\
\text { disorder. }\end{array}$ & 266 & $7-17$ & 118 & $7-17$ \\
\hline $\begin{array}{l}\text { Lieb et al. } \\
(2002)[31]\end{array}$ & & $\begin{array}{l}\text { Alcohol depend- } \\
\text { ence or abuse } \\
\text { (DSM-IV) }\end{array}$ & Community sample & & $\begin{array}{l}\text { Community sample } \\
\text { without alcohol use } \\
\text { disorders }\end{array}$ & $\begin{array}{l}1 \text { AD parent: } \\
469 \\
\text { both AD } \\
\text { parents: } 74\end{array}$ & $14-24$ & 1,866 & $14-24$ \\
\hline $\begin{array}{l}\text { Wilens et al. } \\
(2002)[23]\end{array}$ & $\begin{array}{l}16 \\
14\end{array}$ & $\begin{array}{l}\text { Opioid dependence } \\
\text { (DSM-III-R) } \\
\text { AD } \\
\text { (DSM-III-R) }\end{array}$ & Outpatients & 66 & $\begin{array}{l}\text { Employees of a hospital } \\
\text { without drug or alcohol } \\
\text { use disorders }\end{array}$ & $\begin{array}{l}22 \\
22\end{array}$ & $\begin{array}{l}11.4 \\
6-18 \\
11.4 \\
6-18\end{array}$ & 139 & $\begin{array}{l}11.6 \\
6-18\end{array}$ \\
\hline $\begin{array}{l}\text { Clark et al. } \\
(2004)[25]\end{array}$ & 294 & $\begin{array}{l}\text { Drug dependence } \\
\text { or abuse } \\
\text { (DSM-III-R) }\end{array}$ & $\begin{array}{l}\text { Treated subjects, } \\
\text { advertisement }\end{array}$ & 319 & $\begin{array}{l}\text { Advertisement. No adult } \\
\text { Axis-I disorder. }\end{array}$ & 553 & $\begin{array}{l}10.7 \\
6-14\end{array}$ & 614 & $\begin{array}{l}10.9 \\
6-14\end{array}$ \\
\hline $\begin{array}{l}\text { Hill et al. } \\
(2008)[24]\end{array}$ & & $\begin{array}{l}\text { AD } \\
\text { (DSM-III) } \\
\text { Secondary drug } \\
\text { dependence in either } \\
\text { parent (DSM-III) }\end{array}$ & $\begin{array}{l}\text { Members of multiplex families } \\
\text { with high risk for AD ( } 2 \text { same- } \\
\text { sex siblings with AD of whom } \\
1 \text { was treated) }\end{array}$ & & $\begin{array}{l}\text { Volunteers consisting of } \\
\text { same-sex sibling pairs } \\
\text { without substance } \\
\text { dependence }\end{array}$ & $\begin{array}{r}124 \\
182 \\
50 \\
70\end{array}$ & $\begin{array}{l}8-11 \\
12-18 \\
8-11 \\
12-18\end{array}$ & $\begin{array}{r}93 \\
156 \\
\\
75 \\
129\end{array}$ & $\begin{array}{l}8-11 \\
12-18 \\
8-11 \\
12-18\end{array}$ \\
\hline $\begin{array}{l}\text { Diaz et al. } \\
(2008)[32]\end{array}$ & & $\begin{array}{l}\text { AD } \\
(\mathrm{DSM}-\mathrm{IV})\end{array}$ & Treated subjects & & $\begin{array}{l}\text { Parents of control } \\
\text { children recruited in } \\
\text { schools, no alcohol }\end{array}$ & 371 & $\begin{array}{l}12.5 \\
6-17\end{array}$ & 147 & $\begin{array}{l}12.2 \\
6-17\end{array}$ \\
\hline $\begin{array}{l}\text { Marmorstein } \\
\text { et al. } \\
(2009)[33]\end{array}$ & $\begin{array}{r}270 \\
50\end{array}$ & $\begin{array}{l}\mathrm{AD} \\
\text { (DSM-III-R) } \\
\text { Drug dependence }\end{array}$ & Community sample & 280 & $\begin{array}{l}\text { Community sample } \\
\text { without substance } \\
\text { dependence }\end{array}$ & $\begin{array}{l}540 \\
100\end{array}$ & 17.5 & 560 & 17.5 \\
\hline
\end{tabular}

${ }^{*} \mathrm{p}<0.05 ;{ }^{* *} \mathrm{p}<0.01 ;{ }^{* * *} \mathrm{p}<0.001 . \mathrm{B}=$ Boys; $\mathrm{G}=$ girls. ${ }^{1}$ Conduct disorder. ${ }^{2}$ Alcohol abuse. ${ }^{3}$ Oppositional defiant disorder. ${ }^{4}$ Separation anxiety disorder. ${ }^{5} \mathrm{GAD} /$ overanxious disorder. ${ }^{6}$ Drug abuse. ${ }^{7} \mathrm{AD} .{ }^{8}$ Substance dependence. 


\begin{tabular}{|c|c|c|c|c|c|c|c|c|c|c|c|c|c|}
\hline \multicolumn{14}{|l|}{ Results } \\
\hline \multicolumn{14}{|c|}{ lifetime Axis-I disorders in high-risk (H) and low-risk (L) offspring, \% } \\
\hline \multicolumn{2}{|l|}{ any } & \multicolumn{2}{|c|}{$\mathrm{BPD}$} & \multicolumn{2}{|l|}{ MDD } & \multicolumn{2}{|l|}{ anxiety } & \multicolumn{2}{|l|}{ DBD } & \multicolumn{2}{|c|}{ ADHD } & \multicolumn{2}{|c|}{ substance use } \\
\hline $\mathrm{H}$ & $\mathrm{L}$ & $\mathrm{H}$ & $\mathrm{L}$ & $\mathrm{H}$ & $\mathrm{L}$ & $\mathrm{H}$ & $\mathrm{L}$ & $\mathrm{H}$ & $\mathrm{L}$ & $\mathrm{H}$ & $\mathrm{L}$ & $\mathrm{H}$ & $\mathrm{L}$ \\
\hline 37.8 & 33.3 & & & 11.3 & 9.5 & 22.6 & 21.4 & $5.7^{1}$ & $2.4^{1}$ & 3.8 & 2.4 & $3.8^{2}$ & $0^{2}$ \\
\hline 61 & 49.5 & & & 22 & 15.7 & $30.5^{6}$ & $7.8^{6}$ & $\begin{array}{l}14.6^{1} \\
17.1^{3}\end{array}$ & $\begin{array}{l}9.8^{1} \\
9.8^{3}\end{array}$ & 19.5 & 15.7 & $7.3^{2}$ & $5.9^{2}$ \\
\hline 90.9 & $49.5^{* *}$ & & & 40.9 & 15.7 & $40.9^{6, * *}$ & $7.8^{6}$ & $\begin{array}{l}40.9^{1, * * *} \\
50^{3, * *}\end{array}$ & $\begin{array}{l}9.8^{1} \\
9.8^{3}\end{array}$ & $40.9^{*}$ & 15.7 & $27.3^{2, *}$ & $5.9^{2}$ \\
\hline $60.5^{* *}$ & 28.9 & & & & & 39.5 & 18.4 & 13.2 & 2.6 & 13.2 & 2.6 & & \\
\hline 41.6 & 28.1 & & & 11.7 & 3.5 & 10.4 & 10.5 & $\begin{array}{l}15.6^{1, * *} \\
11.7^{3}\end{array}$ & $\begin{array}{l}0^{1} \\
3.5^{3}\end{array}$ & 13 & 15.8 & $20.5^{* * *}$ & 0 \\
\hline & & & & 6.9 & 23.0 & 20.7 & 30.8 & $27.5^{1}$ & $15.4^{1}$ & 20.6 & 15.4 & 8.3 & 7.7 \\
\hline & & & & 15.8 & 25.0 & 21.1 & 41.7 & $10.5^{1}$ & $8.3^{1}$ & 0 & 4.2 & 0 & 2.2 \\
\hline & & & & 3.0 & 5.6 & 15.2 & 16.7 & $3^{1}$ & $5.6^{1}$ & 15.2 & 5.6 & & \\
\hline & & & & 12.1 & 11.1 & 24.3 & 25.9 & $0^{1}$ & $7.4^{1}$ & 9.1 & 3.7 & & \\
\hline & & & & & & $13.9^{4, *}$ & $\begin{array}{l}5.9^{4} \\
5^{5}\end{array}$ & $14.3^{1}$ & $8.5^{1}$ & 13.2 & 5.9 & $5.6^{2}$ & $\begin{array}{l}7.6^{2} \\
5.16\end{array}$ \\
\hline & & & & & & $9.8^{5, *}$ & $2.5^{5}$ & $6.4^{3}$ & $5.9^{3}$ & & & $1.9^{6}$ & $5.1^{\circ}$ \\
\hline & & & & & & & & & & & & $\begin{array}{l}22^{2} \\
13^{7, * * *} \\
24^{2} \\
16^{7, * *}\end{array}$ & $\begin{array}{l}19^{2} \\
7^{7} \\
19^{2} \\
7^{7} \\
\end{array}$ \\
\hline $59^{* *}$ & 28 & 5 & 0 & $27^{*}$ & 9 & $45^{* *}$ & 15 & $23^{1, * *}$ & $1^{1}$ & $23^{*}$ & 4 & 5 & 1 \\
\hline 41 & & 0 & & $23^{*}$ & & 32 & & $9^{1, *}$ & & 14 & & $18^{*}$ & \\
\hline & & & & $2.4^{*}$ & 0.9 & $8.1^{*}$ & 6.2 & $2.3^{1, * *}$ & $0.3^{1}$ & $7.4^{* *}$ & 4.2 & & \\
\hline 34.7 & 22.6 & 0 & 0 & 3.2 & 0 & $6.5^{4}$ & $6.5^{4}$ & $\begin{array}{l}4.0^{1} \\
12.1^{3, * * *}\end{array}$ & $\begin{array}{l}0^{1} \\
2.2^{3}\end{array}$ & $16.9^{* *}$ & 7.5 & $\begin{array}{l}0 \\
14.3^{* *}\end{array}$ & $\begin{array}{l}0 \\
3.8\end{array}$ \\
\hline & & 0.5 & 0 & $14.8^{* *}$ & 3.8 & $0.5^{4}$ & $1.9^{4}$ & $\begin{array}{l}21.2^{1, * * *} \\
27.5^{3, * * *}\end{array}$ & $\begin{array}{l}3.8^{1} \\
7.1^{3}\end{array}$ & $13.2^{* *}$ & 5.1 & $14.8^{8, *}$ & $6.4^{8}$ \\
\hline $\begin{array}{l}32.0 \\
62.9^{* * *}\end{array}$ & $\begin{array}{l}24.0 \\
32.6\end{array}$ & & & & & & & & & & & & \\
\hline $37.7^{* * *}$ & 12.6 & & & 3.3 & 0 & $\begin{array}{l}4.2^{4} \\
4.8^{5}\end{array}$ & $\begin{array}{l}1.5^{4} \\
0^{5}\end{array}$ & $3.6^{3}$ & $0.7^{3}$ & 11.1 & 4.4 & & \\
\hline & & & & & & & & $\begin{array}{l}24.8^{1} \\
10.5^{3} \\
33^{1} \\
34^{3}\end{array}$ & $\begin{array}{l}4.8^{1} \\
3.3^{3} \\
4.8^{1} \\
3.3^{3}\end{array}$ & $\begin{array}{l}5.7 \\
14\end{array}$ & $\begin{array}{l}0.6 \\
0.6\end{array}$ & $\begin{array}{l}15.4^{7} \\
23^{7}\end{array}$ & $\begin{array}{l}1.1^{7} \\
1.1^{7}\end{array}$ \\
\hline
\end{tabular}


Table 2. Sample characteristics of probands and offspring

\begin{tabular}{|c|c|c|c|c|c|c|}
\hline & $\begin{array}{l}\text { HD } \\
\text { probands } \\
(\mathrm{n}=15)\end{array}$ & $\begin{array}{l}\mathrm{AD} \\
\text { probands } \\
(\mathrm{n}=50)\end{array}$ & $\begin{array}{l}\text { Control } \\
\text { probands } \\
(\mathrm{n}=81)\end{array}$ & Statistic & $\begin{array}{l}\mathrm{p} \\
\text { value }\end{array}$ & $\begin{array}{l}\text { Pairwise } \\
\text { comparison }\end{array}$ \\
\hline \multicolumn{7}{|l|}{ Demographic } \\
\hline Female, \% & 40.0 & 44.0 & 43.2 & $\chi_{2}^{2}=0.08$ & n.s. & \\
\hline Age, mean (SD) & $38.8(4.7)$ & $41.6(6.4)$ & $41.1(7.0)$ & $\mathrm{F}_{2}=1.03$ & n.s. & \\
\hline Married, \% & 33.3 & 66.0 & 80.3 & $\chi_{2}^{2}=14.16$ & $<0.001$ & $\mathrm{AC}$ \\
\hline Number of offspring included, mean (SD) & $1.53(0.74)$ & $2.02(0.96)$ & $1.88(0.75)$ & $\mathrm{F}_{2}=2.03$ & n.s. & \\
\hline \multicolumn{7}{|l|}{ Lifetime Axis-I psychiatric disorders, $\%$} \\
\hline Bipolar disorders & 20.0 & 26.0 & 2.5 & $\chi_{2}^{2}=16.75$ & $<0.001$ & $\mathrm{AB}$ \\
\hline Major depressive disorders & 40.0 & 44.0 & 17.3 & $\chi_{2}^{2}=11.80$ & 0.002 & $\mathrm{~B}$ \\
\hline Any anxiety disorders & 6.7 & 44.0 & 9.9 & $\chi_{2}^{2}=22.10$ & $<0.0001$ & $\mathrm{BC}$ \\
\hline Antisocial personality & 20.0 & 8.0 & 1.2 & FET & $<0.01$ & A \\
\hline Childhood disruptive behavioral disorders & 26.7 & 14.3 & 4.9 & $\chi_{2}^{2}=7.68$ & $<0.05$ & A \\
\hline \multirow[t]{2}{*}{ ADHD } & 0.0 & 8.0 & 2.5 & FET & n.s. & \\
\hline & $\begin{array}{l}\text { Offspring of } \\
\text { HD probands } \\
(\mathrm{n}=23)\end{array}$ & $\begin{array}{l}\text { Offspring of } \\
\text { AD probands } \\
(\mathrm{n}=101)\end{array}$ & $\begin{array}{l}\text { Offspring } \\
\text { of controls } \\
(\mathrm{n}=152)\end{array}$ & Statistic & $\begin{array}{l}\mathrm{p} \\
\text { value }\end{array}$ & $\begin{array}{l}\text { Pairwise } \\
\text { comparison }\end{array}$ \\
\hline \multicolumn{7}{|l|}{ Demographic } \\
\hline Girls, \% & 47.8 & 47.5 & 46.7 & $\chi_{2}^{2}=0.02$ & n.s. & \\
\hline Age, mean (SD) & $11.3(3.46)$ & $11.8(3.37)$ & $11.3(3.33)$ & $\mathrm{F}_{2}=0.61$ & n.s. & \\
\hline Socioeconomic status in the family & $3.3(0.7)$ & $3.0(1.0)$ & $3.5(1.1)$ & $\mathrm{F}_{2}=7.91$ & $<0.001$ & $\mathrm{~B}$ \\
\hline Living with both biological parents, $\%$ & 43.5 & 48.5 & 77.0 & $\chi_{2}^{2}=25.74$ & $<0.0001$ & $\mathrm{AB}$ \\
\hline Exposure to traumatic events, $\%$ & 31.8 & 23.0 & 12.4 & $\chi_{2}^{2}=7.46$ & 0.02 & $\mathrm{AB}$ \\
\hline Directly interviewed, \% & 95.7 & 86.1 & 95.4 & $x_{2}^{2}=7.54$ & $<0.05$ & B \\
\hline
\end{tabular}

FET = Fisher's exact test; $\mathrm{A}=\mathrm{HD}$ probands vs. controls; $\mathrm{B}=\mathrm{AD}$ probands vs. controls; $\mathrm{C}=\mathrm{HD}$ probands vs. $\mathrm{AD}$ probands; $\mathrm{ADHD}=$ attention deficit hyperactivity disorder.

Table 3. Sample characteristics of co-parents

\begin{tabular}{|c|c|c|c|c|c|c|}
\hline \multirow[t]{2}{*}{ Characteristic } & \multicolumn{3}{|l|}{ Spouse of } & \multirow[t]{2}{*}{ Statistic } & \multirow{2}{*}{$\begin{array}{l}\mathrm{p} \\
\text { value }\end{array}$} & \multirow{2}{*}{$\begin{array}{l}\text { Pairwise } \\
\text { comparison }\end{array}$} \\
\hline & $\begin{array}{l}\text { HD proband } \\
(\mathrm{n}=16)\end{array}$ & $\begin{array}{l}\text { AD proband } \\
(\mathrm{n}=57)\end{array}$ & $\begin{array}{l}\text { controls } \\
(\mathrm{n}=85)\end{array}$ & & & \\
\hline \multicolumn{7}{|l|}{ Demographic } \\
\hline Female, \% & 56.3 & 54.4 & 57.7 & $\chi_{2}^{2}=0.14$ & n.s. & \\
\hline Age, mean (SD) & $41.3(4.8)$ & $41.3(5.6)$ & $41.7(7.3)$ & $\mathrm{F}_{2}=0.07$ & n.s. & \\
\hline Interviewed, \% & 31.3 & 49.1 & 67.1 & $\chi_{2}^{2}=9.28$ & 0.01 & $\mathrm{AB}$ \\
\hline \multicolumn{7}{|l|}{ Lifetime Axis-I psychiatric disorders, \% } \\
\hline Any disorder & 46.2 & 46.9 & 46.3 & $\chi_{2}^{2}=0.00$ & n.s. & \\
\hline Bipolar disorders & 7.7 & 10.2 & 6.1 & FET & n.s. & \\
\hline Major depressive disorders & 7.7 & 31.6 & 30.5 & $\chi_{2}^{2}=3.03$ & n.s. & \\
\hline Schizoaffective disorder unipolar & 0.0 & 2.0 & 2.4 & FET & n.s. & \\
\hline Any anxiety disorders & 15.4 & 16.3 & 19.5 & $\chi_{2}^{2}=0.27$ & n.s. & \\
\hline $\mathrm{AD}$ & 23.1 & 10.2 & 1.2 & FET & 0.003 & $\mathrm{AB}$ \\
\hline Alcohol abuse & 0.0 & 6.1 & 7.3 & FET & n.s. & \\
\hline Drug abuse or dependence & 30.8 & 2.0 & 3.7 & FET & 0.003 & $\mathrm{AC}$ \\
\hline Disruptive behavioral disorders & 12.5 & 5.9 & 1.5 & FET & n.s. & \\
\hline $\mathrm{ADHD}, \%$ & 0.0 & 2.9 & 3.1 & FET & n.s & \\
\hline
\end{tabular}

FET $=$ Fisher's exact test $\mathrm{A}=\mathrm{HD}$ vs. controls; $\mathrm{B}=\mathrm{AD}$ vs. controls; $\mathrm{C}=\mathrm{HD}$ vs. $\mathrm{AD} ; \mathrm{ADHD}=$ attention deficit hyperactivity disorder. 
Table 4. Lifetime prevalence of psychiatric disorders in all offspring

\begin{tabular}{|c|c|c|c|c|c|c|}
\hline \multirow[t]{2}{*}{ Offspring disorders } & \multicolumn{6}{|c|}{ All offspring $(\mathrm{n}=276)$} \\
\hline & $\begin{array}{l}\text { HD proband } \\
(\mathrm{n}=23)\end{array}$ & $\begin{array}{l}\text { AD proband } \\
(\mathrm{n}=101)\end{array}$ & $\begin{array}{l}\text { controls } \\
(n=152)\end{array}$ & $x_{2}{ }^{2}$ & $\mathrm{p}$ & $\begin{array}{l}\text { pairwise } \\
\text { comparisons }\end{array}$ \\
\hline Any disorder & 60.9 & 63.4 & 42.1 & 11.9 & 0.003 & $\mathrm{~B}$ \\
\hline Any mood disorder & 21.7 & 29.7 & 17.1 & 7.9 & n.s. & \\
\hline Any depression & 17.4 & 25.7 & 17.1 & 2.9 & n.s. & \\
\hline Any MDD & 13.0 & 15.8 & 11.18 & 1.2 & n.s. & \\
\hline MDD recurrent & 8.7 & 5.0 & 0.7 & FET & 0.01 & $\mathrm{AB}$ \\
\hline MDD single & 4.4 & 9.9 & 8.5 & FET & n.s. & \\
\hline SAD & 0.0 & 3.0 & 0.7 & FET & n.s. & \\
\hline Dysthymia & 0.0 & 1.0 & 2.6 & FET & n.s. & \\
\hline Subthreshold depression & 4.4 & 6.9 & 5.9 & 0.2 & n.s. & \\
\hline Any BPD & 4.4 & 4.0 & 0.0 & FET & 0.02 & $\mathrm{~B}$ \\
\hline BPD I or II, SAM & 4.4 & 3.0 & 0.0 & FET & 0.03 & \\
\hline Subthreshold BPD & 0.0 & 1.0 & 0.0 & FET & n.s. & \\
\hline Any anxiety disorder & 34.8 & 43.6 & 25.7 & 8.8 & 0.01 & $\mathrm{~B}$ \\
\hline Separation anxiety & 26.1 & 32.7 & 18.4 & 6.7 & 0.03 & $\mathrm{~B}$ \\
\hline GAD & 0.0 & 3.0 & 0.0 & FET & n.s. & \\
\hline Panic disorder & 4.4 & 1.0 & 2.0 & FET & n.s. & \\
\hline Agoraphobia & 0.0 & 4.0 & 3.3 & FET & n.s. & \\
\hline Social phobia & 13.0 & 15.8 & 9.9 & 2.0 & n.s. & \\
\hline PTSD & 0.0 & 2.0 & 0.7 & FET & n.s. & \\
\hline Disruptive disorders & 13.0 & 13.8 & 7.9 & 2.5 & n.s. & \\
\hline ADHD & 21.7 & 8.9 & 5.9 & 6.6 & 0.03 & A \\
\hline Alcohol or cannabis ab/dep & 17.4 & 5.0 & 2.0 & FET & 0.01 & $\mathrm{~A}$ \\
\hline Alcohol ab/dep & 8.7 & 4.0 & 2.0 & FET & n.s. & \\
\hline Cannabis ab/dep & 17.4 & 3.0 & 1.3 & FET & 0.002 & $\mathrm{AC}$ \\
\hline Alcohol consumption & 50.0 & 42.5 & 34.7 & 2.7 & n.s. & \\
\hline Cannabis consumption & 13.6 & 9.2 & 2.1 & FET & 0.01 & $\mathrm{AB}$ \\
\hline
\end{tabular}

FET $=$ Fisher's exact tests; $\mathrm{A}=\mathrm{HD}$ vs. controls; $\mathrm{B}=\mathrm{AD}$ vs. controls; $\mathrm{C}=\mathrm{HD}$ vs. $\mathrm{AD} ; \mathrm{MDD}=$ major depressive disorder; $\mathrm{SAD}=$ unipolar schizoaffective disorder; $\mathrm{BPD}=$ bipolar disorder; GAD = generalized anxiety disorder; PTSD = post-traumatic stress disorder; $\mathrm{ADHD}=$ attention deficit hyperactivity disorder; $\mathrm{ab} / \mathrm{dep}=$ abuse or dependence.

Nearly $60 \%$ of these $86 \%$ of co-parents were directly interviewed, with a higher proportion among those of controls as compared to those of $\mathrm{AD}$ or $\mathrm{HD}$ probands (table 3). Co-parents did not differ by age or sex.

Regarding psychopathology, spouses of both HD and $\mathrm{AD}$ probands had higher rates of AD than those of controls. Moreover, spouses of heroin addicts exhibited drug abuse or dependence more frequently than the other coparents.

\section{Sociodemographic Characteristics and}

\section{Psychopathology in Children}

Ninety-one percent of offspring accepted the direct diagnostic interview, with a lower proportion in children of AD probands as compared to those of controls (table 2). Offspring did not differ in age or sex by parental diagnos- tic status. However, offspring of psychiatric probands were less likely to be living with both parents but more likely to report exposure to traumatic events than those of controls. In addition, the socioeconomic status of the family was lower for children of $\mathrm{AD}$ probands than for children of controls.

Table 4 presents the lifetime prevalence rates of psychiatric disorders across the three groups of offspring. The offspring of both $\mathrm{HD}$ and $\mathrm{AD}$ probands revealed increased rates of recurrent MDD, whereas only the children of $\mathrm{AD}$ probands exhibited any Axis-I psychiatric disorder and any BPD more frequently than those of comparison probands. The offspring of AD probands were also found to be at an increased risk of any anxiety disorder and separation anxiety disorder compared to children of controls. 
Table 5. Adjusted OR (95\% CI) for psychiatric disorders in offspring of parents with SUD $^{1}$

\begin{tabular}{|c|c|c|}
\hline & HD in proband & $\mathrm{AD}$ in proband \\
\hline Any disorder & $1.6(0.7 ; 3.8)$ & $1.1(0.6 ; 2.2)$ \\
\hline Any mood disorder & $1.1(0.4 ; 3.1)$ & $1.0(0.5 ; 2.2)$ \\
\hline Any depression & $0.9(0.3 ; 2.8)$ & $1.0(0.4 ; 2.3)$ \\
\hline Any MDD & $1.3(0.3 ; 5.3)$ & $1.4(0.4 ; 4.5)$ \\
\hline MDD recurrent & $8.1(1.2 ; 55.3)^{*}$ & $7.9(1.2 ; 49.4)^{*}$ \\
\hline MDD single & $0.5(0.1 ; 4.6)$ & $0.8(0.2 ; 2.8)$ \\
\hline Any anxiety disorder & $1.1(0.4 ; 2.9)$ & $1.0(0.5 ; 2.1)$ \\
\hline Separation anxiety & $0.9(0.3 ; 2.9)$ & $0.7(0.3 ; 1.5)$ \\
\hline Social phobia & $1.2(0.3 ; 5.3)$ & $1.3(0.5 ; 3.8)$ \\
\hline Disruptive disorders & $1.2(0.4 ; 4.1)$ & $1.3(0.6 ; 2.9)$ \\
\hline $\mathrm{ADHD}$ & $4.2(1.2 ; 14.2)^{*}$ & $0.5(0.1 ; 2.0)$ \\
\hline Alcohol or cannabis ab/dep & $16.1(1.6 ; 166.6)^{*, 2}$ & $0.6(0.0 ; 6.5)^{2}$ \\
\hline Alcohol consumption & $3.1(1.4 ; 6.9)^{*, 2}$ & $0.8(0.4 ; 1.6)^{2}$ \\
\hline Drug consumption & $5.3(0.3 ; 103.5)^{2}$ & $2.2(0.2 ; 25.4)^{2}$ \\
\hline \multicolumn{3}{|c|}{$\begin{array}{l}\text { MDD = Major depressive disorder; } \mathrm{ADHD}=\text { attention deficit hyperactivity disorder; } \\
\mathrm{ab} / \mathrm{dep}=\text { abuse or dependence. }{ }^{*} \mathrm{p}<0.05 . \\
{ }^{1} \text { Adjusting for offspring age and sex, family socioeconomic status and proband co- } \\
\text { morbidity(MDD, bipolar disorders, anxiety disorders, disruptive disorders, and ADHD). } \\
{ }^{2} \text { Not adjusted for proband's ADHD. }\end{array}$} \\
\hline
\end{tabular}

Table 6. Psychopathology in offspring by SUD diagnoses of the 2 parents

\begin{tabular}{|c|c|c|c|c|c|c|c|}
\hline \multirow{2}{*}{$\begin{array}{l}\text { Disorder in offspring } \\
\text { SUD }\end{array}$} & \multirow{4}{*}{$\begin{array}{l}\text { Disorder in co-parent } \\
\text { Drug ab/dep } \\
\text { Alcohol ab/dep } \\
\text { No SUD }\end{array}$} & \multicolumn{2}{|c|}{ HD proband } & \multicolumn{2}{|c|}{$\mathrm{AD}$ proband } & \multicolumn{2}{|c|}{ Control proband } \\
\hline & & $50 \%$ & $(3 / 6)$ & $0 \%$ & $(0 / 2)$ & $0 \%$ & $(0 / 4)$ \\
\hline & & - & $(0 / 0)$ & $13.3 \%$ & $(2 / 15)$ & $0 \%$ & $(0 / 14)$ \\
\hline & & $7.7 \%$ & $(1 / 13)$ & $4.2 \%$ & $(3 / 72)$ & $2.3 \%$ & $(3 / 127)$ \\
\hline \multirow[t]{3}{*}{ Alcohol consumption } & Drug ab/dep & $66.7 \%$ & $(4 / 6)$ & $0 \%$ & $(0 / 2)$ & $75.0 \%$ & $(3 / 4)$ \\
\hline & Alcohol ab/dep & - & $(0 / 0)$ & $27.3 \%$ & $(3 / 11)$ & $38.5 \%$ & $(5 / 13)$ \\
\hline & No SUD & $33.3 \%$ & $(4 / 12)$ & $45.5 \%$ & $(30 / 66)$ & $33.3 \%$ & $(41 / 123)$ \\
\hline \multirow[t]{3}{*}{ ADHD } & Drug ab/dep & $16.7 \%$ & $(1 / 6)$ & $50 \%$ & $(1 / 2)$ & $0 \%$ & $(0 / 4)$ \\
\hline & Alcohol ab/dep & - & $(0 / 0)$ & $13.3 \%$ & $(2 / 15)$ & $0 \%$ & $(0 / 14)$ \\
\hline & No SUD & $23.1 \%$ & $(3 / 13)$ & $5.6 \%$ & $(4 / 72)$ & $6.2 \%$ & $(8 / 130)$ \\
\hline
\end{tabular}

SUD = Substance use disorders; $\mathrm{ab} / \mathrm{dep}=$ abuse or dependence; ADHD = attention deficit hyperactivity disorder.

Moreover, children of HD probands had significantly increased rates of ADHD and alcohol or cannabis abuse/ dependence than children of controls. These children also exhibited higher rates of cannabis abuse/dependence than children of alcoholic probands, whereas the offspring of probands with either type of substance dependence reported cannabis consumption more frequently than the offspring of controls.

Table 5 provides the adjusted odds ratios (OR) derived from the generalized multilevel mixed models assessing the risk of psychopathology in children. These models, which adjusted for demographic characteristics in the families and comorbid disorders in probands, confirmed strong associations between $\mathrm{AD}$ and $\mathrm{HD}$ in probands and recurrent MDD in children. Moreover, HD in probands was associated with ADHD and SUD in offspring. Due to the rareness of alcohol and cannabis abuse/dependence in children, it was not possible to apply separate models for these specific substance disorders. However, alcohol consumption was significantly associated with HD in probands. 


\section{Effect of Co-Parental Disorders}

Table 6 provides the rates of disorders in children by substance disorder status of the 2 parents. In co-parents, drug disorders (heroin, cocaine, marijuana abuse and dependence) were combined. The table was restricted to disorders significantly associated with the proband's SUD according to the generalized multilevel mixed models presented in table 5. As no child of a co-parent with drug or alcohol disorders exhibited recurrent MDD, this diagnosis could not be included in the table. Although the absolute numbers of affected children were small, it is remarkable that 3 out of 6 children with both parents affected by drug disorders met criteria for SUD. Moreover, 2 out of the 3 children who did not develop SUD in these families were less than 10 years old. Four ( 2 of them developed SUD) out of the 6 children with 2 parents with drug use disorders were no longer living with the 2 parents, whereas this was true for only one third of the offspring of families of HD probands and a co-parent without SUD.

With respect to alcohol consumption or ADHD in offspring, the rates of these disorders were not particularly high in children of parents who were concordantly affected by SUD.

Generalized multilevel mixed models revealed significant interactions between HD in probands and drug disorders in co-parents as well as between AD in probands and alcohol disorders in co-parents to affect the risk of SUD in children (with adjustment for comorbid mood and anxiety disorders in the two parents as well as ADHD and DBD in probands):

Odds of SUD children $=-0.269+$ (effects of covariates)

$+0.080 \times \mathrm{HD}_{\text {prob }}-0.003 \times \mathrm{AD}_{\text {prob }}-0.007 \times \mathrm{HD}_{\text {co-par }}$

$-0.009 \times \mathrm{AD}_{\text {co-par }}+0.333 \times \mathrm{HD}_{\text {prob }} \times \mathrm{HD}_{\text {co-par }}$

$+0.151 \times \mathrm{AD}_{\text {prob }} \times \mathrm{AD}_{\text {co-par }}$

The drug-drug and alcohol-alcohol interactions were significant at $\mathrm{p}=0.0066$ and $\mathrm{p}=0.0335$, respectively. This means that the combined effect of 2 affected parents on the risk for a child was higher than what would be expected for the product of the effect of each affected parent. These interactions remained statistically significant after adjustment for the family factor 'living with both parents' ( $\mathrm{p}=0.0067$ and $\mathrm{p}=0.0338$, respectively) as well as after adjustment for exposure to traumatic events $(\mathrm{p}=$ 0.0015 and $\mathrm{p}=0.0006$, respectively).

With respect to alcohol consumption and ADHD in children, the models did not reveal significant interactions between parental substance use diagnoses. Moreover, after introducing the main effects of co-parental disorders into the models, the association between HD in probands and alcohol consumption $(\mathrm{OR}=2.2,95 \% \mathrm{CI}$ 0.7-6.7) or ADHD (OR $=3.4,95 \%$ CI 0.6-18.8) in children no longer reached statistical significance.

Regarding the risk of recurrent MDD in offspring, which was not associated with drug or alcohol disorders in co-parents, we could establish that the elevated rates of this disorder in offspring of $\mathrm{HD}$ and $\mathrm{AD}$ probands was not due to mood disorders in co-parents or the family factor 'living with both parents' given that the model adjusting for these two factors still revealed significant associations between the proband's HD or AD and the offspring's recurrent $\mathrm{MDD}(\mathrm{OR}=9.8,95 \% \mathrm{CI} 1.5-63.3$ and $\mathrm{OR}=$ $10.7,95 \%$ CI 1.1-106.5, respectively). The models including the child's exposure to traumatic events still provided similar results for the association between $\mathrm{HD}$ or $\mathrm{AD}$ in probands and the offspring's recurrent $\mathrm{MDD}(\mathrm{OR}=5.9$, 95\% CI 0.7-51.0 and OR $=16.4,95 \%$ CI 2.0-136.0, respectively), although the association between the proband's HD and the child's recurrent MDD failed to reach statistical significance.

\section{Discussion}

The most salient findings of the present study are: (1) children of parents with both HD or AD have approximately 8 times elevated rates of recurrent MDD; (2) children of parents with HD but not with $\mathrm{AD}$ are at a more than 3 times increased risk of ADHD and approximately 16 times increased risk of SUD than children of controls after adjustment for demographic characteristics and the proband's comorbid disorders, and (3) parental concordance for drug and alcohol disorders is likely to be associated with a particularly high risk of early SUD in offspring.

Although we did not observe a significantly elevated overall risk of Axis-I mental disorders in our young sample after adjustment for demographic variables in children and comorbid disorders in probands, our findings of an elevated risk of specific mental disorders in these offspring at high risk support and extend those of the majority of previous research in this field $[21,23-25,27,28$, 30-32]. Indeed, several but not all studies have documented increased rates of mood disorders or internalizing disorders in the offspring of heroin addicts [23-25] as well as in offspring of probands with $\mathrm{AD}[23,24]$. However, the largely increased rate of the recurrent depression subtype regardless of the type of proband's SUD has not been demonstrated before. Interestingly and in contrast 
to our expectations, we did not find higher rates of psychopathology in the offspring of HD probands as compared to that of alcoholic probands with the exception of ADHD and SUD. The significantly elevated rate of $\mathrm{ADHD}$ in the offspring of $\mathrm{HD}$ but not $\mathrm{AD}$ probands corroborates a similar finding in the study of Wilens et al. [23].

In contrast to most previous research, we could control for the effects of comorbid disorders in probands and co-parents, given the systematic collection of diagnostic information on the 2 parents. This allowed us to show that the elevated risk of recurrent MDD in the high-risk children was not due to the direct parent-offspring transmission of these disorders. This increased risk could be attributable either to particular environmental circumstances in the families of dependent probands or genetic factors shared by SUD and MDD. However, although the factors 'living with both biological parents' and the child's exposure to traumatic events, which can be considered as indicators of the child's exposure to stress, were found to be associated with the proband's SUD, the adjustment for these factors in our models only slightly affected the results. In addition, it has been recently shown that the occurrence of early childhood disorders, such as MDD, is likely to increase the risk of the subsequent development of SUD [50].

Regarding the risk of $\mathrm{ADHD}$, the strong association between this disorder in offspring and HD in probands was no longer significant after adjusting for co-parental variables. However, the $\mathrm{OR}>3$ together with the large CI is an indicator of insufficient statistical power to accurately establish this association.

As to SUD, the effect of parental concordance on the risk of psychopathology in children is difficult to establish given that offspring of 2 parents with these disorders are rare. In our study, after the inclusion of co-parental variables, our results were compatible with an interaction between SUD in the 2 parents in relation to the risk of these disorders in children. The finding of a particularly high risk for SUD in children of parents concordant for these disorders is in line with previous studies that documented an association between the number of affected parents and psychopathology $[27,28]$ or alcohol consumption [31] in children. Our observation according to which children of concordantly affected parents were more likely to live with only 1 biological parent could indicate that they were exposed to a less stable familial environment. Moreover, having at least 1 non-affected biological parent is likely to have a protective effect on the child. However, the interactions between SUD in the 2 parents remained significant after the adjustment for the factors 'living with both biological parents' and the child's exposure to traumatic events. Alternatively, the high risk of SUD in children of concordantly affected biological parents could be attributable to genetic interaction.

\section{Strengths and Limitations}

In contrast to previous high-risk research on SUD, which rarely included probands with both $\mathrm{AD}$ and $\mathrm{HD}$, the design of the present study allowed us to simultaneously compare the risk of specific DSM-IV disorders in the offspring of probands with both major subtypes of dependences to that of children of a medical comparison group. Moreover, the collection of diagnostic information on the biological co-parent enabled us to examine the association between the SUD of the 2 parents and the risk of mental disorders in offspring. In addition, unlike most previous studies which lumped several drug disorders together, our results are based on a homogeneous proband group of heroin addicts and we were able to adjust for the effects of co-morbid disorders in probands, which are very common in patients with drug or $\mathrm{AD}$.

The results of the present study should be considered in the context of several limitations. First, our offspring sample and in particular that of probands with HD was small and the latter proband group also revealed selection bias with respect to comorbid lifetime ADHD (absence of this disorder in HD probands). Accordingly, conclusions regarding the children of heroin addicts need to be taken with caution. Moreover, the size of the samples in our study was too small to accurately estimate the prevalence of relatively rare disorders in childhood and adolescence such as BPD. Similarly, our small sample size did not allow us to adjust for the effect of rare co-morbid disorders in the co-parents. Second, although probands who agreed to enroll at least 1 of their children in the study and those who did not were found to differ only with respect to the rates of comorbid ADHD among heroin addicts, we have no information on children of the latter probands. It is possible that highly disrupted families, which were most severely affected by the proband's disorder, were less likely to participate. As the offspring in such families are likely to be at a higher risk of psychopathology and malfunctioning, the present study may provide a too optimistic picture of the children of SUD parents. Third, diagnoses of approximately $5 \%$ of the offspring of $\mathrm{HD}$ and control probands as well as approximately $14 \%$ of the offspring of $\mathrm{AD}$ probands were based on family history reports rather than direct inter- 
views. Given the significantly lower proportion of interviewed offspring of probands with $\mathrm{AD}$, the rates of psychopathology in these children could have been underestimated. Fourth, although a lifetime history of mental disorders was collected for $91 \%$ of biological co-parents, we had no direct diagnostic interview and had to rely on family history reports for $43 \%$ of them.

\section{Implications}

The findings of the present study have several potential clinical implications. First, the higher risk of early recurrent depression, SUD and ADHD in the offspring of these families emphasizes the need to pay particular attention to these children. The prompt identification and treatment of early psychopathology in these children may be critical for the prevention of the development of more severe SUD and other mental disorders. Second, the find- ing of an interaction between SUD in the 2 parents to affect the risk of SUD in their children supports the involvement of co-parental disorders in the development of psychopathology in children of AD and HD patients and highlights the need to pay clinical attention not only to the affected proband but also to his/her partner in order to optimize prevention in offspring.

Longitudinal studies that follow high-risk youth through adolescence and early adulthood are necessary to determine to which extent early recurrent depression and SUD are only a temporary phenomenon or a predictor of subsequent severe SUD. Similarly, prospective research would also allow the study of the links between ADHD and early manifestations of mood disorders and the later development of SUD and other adult mental disorders.

\section{References}

1 Johnson JL, Leff M: Children of substance abusers: overview of research findings. Pediatrics 1999;103:1085-1099.

-2 Uchtenhagen A: Heroin-assisted treatment in Switzerland: a case study in policy change. Addiction 2010;105:29-37.

-3 Donovan JE: Adolescent alcohol initiation: a review of psychosocial risk factors. J Adolesc Health 2004;35:529-518.

4 Anderson P, Baumberg B: Alcohol in Europe: a public health perspective. A report for the European Commission, Health and Consumer Protection, Institute of Alcohol Studies, UK, 2006.

-5 Saraceno L, Munafo M, Heron J, Craddock $\mathrm{N}$, van den Bree MB: Genetic and non-genetic influences on the development of co-occurring alcohol problem use and internalizing symptomatology in adolescence: a review. Addiction 2009;104:1100-1121.

-6 Armstrong TD, Costello EJ: Community studies on adolescent substance use, abuse, or dependence and psychiatric comorbidity. J Consult Clin Psychol 2002;70:1224-1239.

-7 Zatonski WA, Sulkowska U, Manczuk M, Rehm J, Boffetta P, Lowenfels AB, La Vecchia C: Liver cirrhosis mortality in Europe, with special attention to Central and Eastern Europe. Eur Addict Res 2010;16:193-201.

-8 Rehm J, Patra J, Gnam WH, SarnocinskaHart A, Popova S: Avoidable cost of alcohol abuse in Canada. Eur Addict Res 2011;17:7279.

$\checkmark 9$ Cotton NS: The familial incidence of alcoholism: a review. J Stud Alcohol 1979;40:89116.
10 Goodwin DW: Alcoholism and genetics. The sins of the fathers. Arch Gen Psychiatry 1985;42:171-174.

11 Begleiter H, Porjesz B: Potential biological markers in individuals at high risk for developing alcoholism. Alcohol Clin Exp Res 1988; 12:488-493.

12 Devor EJ, Cloninger CR: Genetics of alcoholism. Annu Rev Genet 1989;23:19-36.

13 Maier W, Merikangas K: Co-occurrence and contransmission of affective disorders and alcoholism in families. Br J Psychiatry Suppl 1996;30:93-100.

14 Merikangas KR, Stolar M, Stevens DE, Goulet J, Preisig MA, Fenton B, Zhang $\mathrm{H}$, O'Malley SS, Rounsaville BJ: Familial transmission of substance use disorders. Arch Gen Psychiatry 1998;55:973-979.

15 Merikangas KR, Avenevoli S: Implications of genetic epidemiology for the prevention of substance use disorders. Addict Behav 2000; 25:807-820.

16 Preisig M, Fenton BT, Stevens DE, Merikangas KR: Familial relationship between mood disorders and alcoholism. Compr Psychiatry 2001;42:87-95.

17 Merikangas KR, Gelernter CS: Comorbidity for alcoholism and depression. Psychiatr Clin North Am 1990;13:613-632.

18 McGue M: Genes, environment and the etiology of alcoholism; in Zucker R, Boyd G, Howard J (eds): The Development of Alcohol Problems: Exploring the Biopsychosocial Matrix. Monograph No. 26. Rockville, US Department of Health and Human Services, 1994.
19 Rounsaville BJ, Kosten TR, Weissman MM, Prusoff B, Pauls D, Anton SF, Merikangas K: Psychiatric disorders in relatives of probands with opiate addiction. Arch Gen Psychiatry 1991;48:33-42.

20 Stabenau JR: Is risk for substance abuse unitary? J Nerv Ment Dis 1992;180:583-588.

-21 Merikangas KR, Dierker LC, Szatmari P: Psychopathology among offspring of parents with substance abuse and/or anxiety disorders: a high-risk study. J Child Psychol Psychiatry 1998;39:711-720.

22 Merikangas KR, Low NC: The epidemiology of mood disorders. Curr Psychiatry Rep 2004;6:411-421.

23 Wilens TE, Biederman J, Bredin E, Hahesy AL, Abrantes A, Neft D, Millstein R, Spencer TJ: A family study of the high-risk children of opioid- and alcohol-dependent parents. Am J Addict 2002;11:41-51.

24 Hill SY, Shen S, Lowers L, Locke-Wellman J, Matthews AG, McDermott M: Psychopathology in offspring from multiplex alcohol dependence families with and without parental alcohol dependence: a prospective study during childhood and adolescence. Psychiatry Res 2008;160:155-166.

25 Clark DB, Cornelius J, Wood DS, Vanyukov M: Psychopathology risk transmission in children of parents with substance use disorders. Am J Psychiatry 2004;161:685-691.

26 Hill SY, Hruska MA: Childhood psychopathology in families with multigenerational alcoholism. J Am Acad Child Adolesc Psychiatry 1992;31:1024-1030. 
27 Reich W, Earls F, Frankel O, Shayka JJ: Psychopathology in children of alcoholics. J Am Acad Child Adolesc Psychiatry 1993;32:9951002.

28 Hill SY, Muka D: Childhood psychopathology in children from families of alcoholic female probands. J Am Acad Child Adolesc Psychiatry 1996;35:725-733.

-29 Nunes EV, Weissman MM, Goldstein RB, McAvay G, Seracini AM, Verdeli H, Wickramaratne PJ: Psychopathology in children of parents with opiate dependence and/or major depression. J Am Acad Child Adolesc Psychiatry 1998;37:1142-1151.

- 30 Kuperman S, Schlosser SS, Lidral J, Reich W: Relationship of child psychopathology to parental alcoholism and antisocial personality disorder. J Am Acad Child Adolesc Psychiatry 1999;38:686-692.

- 31 Lieb R, Merikangas KR, Hofler M, Pfister H, Isensee B, Wittchen HU: Parental alcohol use disorders and alcohol use and disorders in offspring: a community study. Psychol Med 2002;32:63-78.

-32 Diaz R, Gual A, Garcia M, Arnau J, Pascual F, Canuelo B, Rubio G, de DY, FernandezEire MC, Valdes R, Garbayo I: Children of alcoholics in Spain: from risk to pathology. Results from the ALFIL program. Soc Psychiatry Psychiatr Epidemiol 2008;43:1-10.

33 Marmorstein NR, Iacono WG, McGue M: Alcohol and illicit drug dependence among parents: associations with offspring externalizing disorders. Psychol Med 2009;39: 149-155.

- 34 Dierker LC, Merikangas KR, Szatmari P: Influence of parental concordance for psychiatric disorders on psychopathology in offspring. J Am Acad Child Adolesc Psychiatry 1999;38:280-288.
35 Nurnberger JI Jr, Blehar MC, Kaufmann CA, York-Cooler C, Simpson SG, HarkavyFriedman J, Severe JB, Malaspina D, Reich T: Diagnostic interview for genetic studies. Rationale, unique features, and training NIMH Genetics Initiative. Arch Gen Psychiatry 1994;51:849-859.

36 Preisig M, Fenton BT, Matthey ML, Berney A, Ferrero F: Diagnostic Interview for Genetic Studies (DIGS): inter-rater and test-retest reliability of the French version. Eur Arch Psychiatry Clin Neurosci 1999;249: 174-179.

37 Berney A, Preisig M, Matthey ML, Ferrero F, Fenton BT: Diagnostic Interview for Genetic Studies (DIGS): inter-rater and test-retest reliability of alcohol and drug diagnoses. Drug Alcohol Depend 2002;65:149-158.

38 Orvaschel H, Puig-Antich J, Chambers W, Tabrizi MA, Johnson R: Retrospective assessment of prepubertal major depression with the K-SADS-E. J Am Acad Child Psychiatry 1982;21:392-397.

39 Gammon GD, John K, Rothblum ED, Mullen K, Tischler GL, Weissman MM: Use of a structured diagnostic interview to identify bipolar disorder in adolescent inpatients: frequency and manifestations of the disorder. Am J Psychiatry 1983;140:543-547.

40 Chambers WJ, Puig-Antich J, Hirsch M, Paez P, Ambrosini PJ, Tabrizi MA, Davies M: The assessment of affective disorders in children and adolescents by semistructured interview. Test-retest reliability of the Schedule for Affective Disorders and Schizophrenia for school-age children, present episode version. Arch Gen Psychiatry 1985;42:696-702.

-41 Andreasen NC, Endicott J, Spitzer RL, Winokur G: The family history method using diagnostic criteria. Reliability and validity. Arch Gen Psychiatry 1977;34:1229-1235.

-42 Rougemont-Buecking A, Rothen S, Jeanpretre N, Lustenberger Y, Vandeleur CL, Ferrero F, Preisig M: Inter-informant agreement on diagnoses and prevalence estimates of anxiety disorders: direct interview versus family history method. Psychiatry Res 2008;157: 211-223.
43 Vandeleur CL, Rothen S, Jeanpretre N, Lustenberger Y, Gamma F, Ayer E, Ferrero F, Fleischmann A, Besson J, Sisbane F, Preisig $\mathrm{M}$ : Inter-informant agreement and prevalence estimates for substance use disorders: direct interview versus family history method. Drug Alcohol Depend 2008;92:9-19.

44 Rothen S, Vandeleur CL, Lustenberger Y, Jeanpretre N, Ayer E, Gamma F, Halfon O, Fornerod D, Ferrero F, Preisig M: Parentchild agreement and prevalence estimates of diagnoses in childhood: direct interview versus family history method. Int J Methods Psychiatr Res 2009;18:96-109.

45 Leckman JF, Sholomskas D, Thompson WD, Belanger A, Weissman MM: Best estimate of lifetime psychiatric diagnosis: a methodological study. Arch Gen Psychiatry 1982;39: 879-883.

46 Angst J, Gamma A, Benazzi F, Ajdacic V, Eich D, Rossler W: Toward a re-definition of subthreshold bipolarity: epidemiology and proposed criteria for bipolar-II, minor bipolar disorders and hypomania. J Affect Disord 2003;73:133-146.

47 Hollingshead AB: Four Factor Index of Social Status. New Haven, Yale University Press, 1975.

48 Liang KY, Zeger SL: Longitudinal data analysis using generalized linear models. Biometrika 1986;73:13-22.

-49 Ringlever L, Otten R, de Leeuw RN, Engels RC: Effects of parents' education and occupation on adolescent smoking and the mediating role of smoking-specific parenting and parent smoking. Eur Addict Res 2011;17:5563.

50 Hill SY, Tessner KD, McDermott MD: Psychopathology in offspring from families of alcohol-dependent female probands: a prospective study. J Psychiatr Res 2011;45:285294 